\title{
UNA NUEVA ESPECIE DE METAPHYCUS MERCET (HYMENOPTERA: ENCYRTIDAE) DE MÉXICO Y CLAVE DE ESPECIES DEL GÉNERO QUE PARASITAN MOSQUITAS BLANCAS (HEMIPTERA: ALEYRODIDAE) EN LA REGIÓN NEOTROPICAL
}

\author{
Svetlana N. Myartseva y Enrique Ruíz Cancino \\ División de Estudios de Postgrado e Investigación, UAM Agronomía y Ciencias, Universidad \\ Autónoma de Tamaulipas, 87149 Cd. Victoria, Tamaulipas, MÉXICO \\ E mail: smyartse@uat.edu.mx; eruiz@uat.edu.mx
}

Myartseva, S. N. \& E. Ruíz-Cancino. 2010. Una nueva especie de Metaphycus Mercet (Hymenoptera: Encyrtidae) de México y clave de especies del género que parasitan mosquitas blancas (Hemiptera:

Aleyrodidae) en la región neotropical. Acta Zoológica Mexicana (n.s.), 26(1):17-24.

RESUMEN. Se describe a Metaphycus cerealis n. sp., un parasitoide de la mosquita blanca Aleurocybotus occiduus (Russell) del Estado de Sinaloa, México. Se elaboró la clave para las especies de Metaphycus que parasitan mosquitas blancas en la Región Neotropical.

Palabras clave: Metaphycus, mosquitas blancas, Latinoamérica.

Myartseva, S. N. \& E. Ruíz-Cancino. 2010. A new species of Metaphycus Mercet (Hymenoptera: Encyrtidae) from Mexico and key to the species of the genera parasitizing whiteflies (Hemiptera:

Aleyrodidae) in the Neotropical region. Acta Zoológica Mexicana (n.s.), 26(1):17-24.

ABSTRACT. Metaphycus cerealis sp. nov., a parasitoid of the whitefly Aleurocybotus occiduus (Russell) from the State of Sinaloa, Mexico, is described. A key to species of Metaphycus parasitizing whiteflies in the Neotropic Region is given.

Key words: Metaphycus, whiteflies, Latin America.

\section{INTRODUCCIÓN}

El género Metaphycus Mercet cuenta con más de 400 especies descritas a nivel mundial. Más de 200 especies se encuentran en la región Neotropical (Noyes 2004). Son endoparasitoides primarios de escamas (Hemiptera: Coccidae, Diaspididae). Recientemente, algunas especies de este género han sido obtenidas también de mosquitas blancas (Hemiptera: Aleyrodidae) en los neotrópicos.

Polaszek et al. (1992) reportaron especies no identificadas de Metaphycus que emergieron de ninfas de la mosquita blanca Bemisia tabaci Gennadius; dicho género no había sido registrado antes como parasitoide de Aleyrodidae. Posteriormente, Metaphycus omega Noyes, 2004 se reportó como parasitoide de mosquitas blancas

Recibido: 02/10/2008; aceptado: 12/10/2009. 
en Brasil, Costa Rica, Ecuador, Guyana, Paraguay y Trinidad. Otras especies se han reportado atacando aleyródidos: $M$. troas Noyes, 2004 de Costa Rica y México, $M$. acapulcus Myartseva \& Ruíz, 2003 y M. aleyrodis (Myartseva \& Ruíz 2002) fueron descritas de México, y M. zdeneki Noyes \& Lozada, 2005 descrita de Perú (Myartseva \& Ruíz Cancino 2002, 2003, 2004; Noyes 2004; Noyes \& Lozada 2005). Sólo algunas especies de Metaphycus atacan mosquitas blancas (Noyes 2004) y forman un grupo muy especializado que no está muy emparentado con las otras especies del género que parasitan Coccoidea.

En el estado de Sinaloa, México, en 2007 se obtuvieron algunos parasitoides de una mosquita blanca que infesta diversas gramíneas (Ortega et al. 2008). Dicha especie de aleyródido, Aleurocybotus occiduus (Russell), ocurre en Estados Unidos (continental), Hawaii y Perú (Noyes 2007) y es un nuevo registro para México. En el presente artículo se describe una nueva especie de Metaphycus, la cual fue obtenida de Aleurocybotus occiduus en México, se incluyen datos sobre los hospederos de Metaphycus spp. y se elaboró la clave para identificar las especies de Metaphycus que atacan mosquitas blancas en la región Neotropical.

\section{MATERIAL Y MÉTODOS}

Durante julio-noviembre 2007, en Guasave, Sinaloa, México, se encontraron numerosas colonias de la mosquita blanca Aleurocybotus occiduus (Russell) en muchas gramíneas silvestres y cultivadas, incluyendo caña de azúcar Saccharum officinarum L., sorgo Sorghum vulgare Pers. y maíz Zea mays L. En junio de 2008 se recibió el envío de parasitoides emergidos de las ninfas de la mosquita blanca para su identificación. Los parasitoides fueron conservados en alcohol al $70 \%$.

Para estudiar la morfología e identificación de los parasitoides, algunos especímenes fueron disectados y montados en portaobjetos con bálsamo de Canadá. En la identificación de familias, géneros y especies de las avispas parasíticas, se usaron claves especializadas (Noyes et al. 1997; Noyes 2004; Myartseva \& Ruíz Cancino 2004; Myartseva 2006). La especie de mosquita blanca fue identificada por el Dr. Vicente E. Carapia como Aleurocybotus occiduus (Russell), la cual fue reportada como un nuevo registro para México por Ortega et al. (2008).

\section{RESULTADOS Y DISCUSIÓN}

Descripción de la nueva especie Metaphycus cerealis $\mathrm{n}$. sp.

Figs. 1-3

Material tipo. Holotipo 우 (montada en portaobjetos): México, Sinaloa, Guasave, Ejido A. Ruíz Cortínez, ex Aleurocybotus occiduus (Russell) en Saccharum sp., VIIXI. 2007 (G. Vejar-Cota). Paratipos: 5 \&, 2 ๙ - mismos datos que el holotipo. 
El holotipo de la nueva especie está depositado en el Entomological Museum, University of California (UCR), en Riverside, CA, EUA; los paratipos: 2 ㅇ, 2 o -en UCR, y 3 q- en el Museo de Insectos de la UAM Agronomía y Ciencias - UAT, en Ciudad Victoria, Tamaulipas, México.

Descripción. Hembra. Longitud del cuerpo: 0.9-1.0 mm.

Coloración. Cabeza amarilla; antenas amarillo claro, maza ligeramente ahumada. Mesosoma amarillo. Patas amarillo blancuzco. Alas anteriores ligeramente ahumadas debajo de la vena marginal. Metasoma amarillo, ápices ahumados en la tercera válvula y las placas de los cercos.

Estructura. Mandíbula con dos dientes agudos, el diente superior más largo (Fig. 1). Palpos labiales y maxilares de 1 y 2 segmentos, respectivamente. Antenas (Fig. 2) insertadas ligeramente arriba del margen bucal. Radícula cerca de 2 veces tan larga como ancha. Escapo cilíndrico, 5.0-6.5 veces tan largo como ancho. Pedicelo cerca de 2 veces tan largo como ancho y más corto que los primeros 3 segmentos funiculares juntos. Segmentos funiculares 1-4 subiguales en longitud, segmentos 1 y 2 cuadrados, segmentos 3 y 4 ligeramente transversales; segmentos 5 y 6 ligeramente más largos que el segmento precedente pero el segmento 5 ligeramente más largo que ancho, cada uno con una sensila. Setas del funículo más largas que la anchura de los segmentos. Maza cerca de 2.4 veces tan larga como ancha y tan larga como los 4 segmentos funiculares precedentes juntos. Mesoescudo con notalos indicados lateralmente por líneas superficiales. Ala anterior cerca de 3 veces tan larga como la anchura alar máxima. Setas del disco muy delgadas y cortas entre la parte ahumada y el tercio apical. Vena marginal puntiforme. Espuela de la tibia media más o menos tan larga como el basitarso. Ovipositor ligeramente expuesto, ligeramente más largo que la tibia media; tercera válvula cerca de 0.3 veces tan larga como el segundo valvifer.

Macho. Longitud del cuerpo: 0.8-0.9 mm. Similar en coloración a la hembra pero con el flagelo antenal ahumado. Escapo antenal cerca de 4 veces tan largo como ancho (Fig. 3). Pedicelo más o menos tan largo como los primeros dos segmentos funiculares juntos. Todos los segmentos flagelares subcuadrados, ligeramente agrandados hacía el ápice; segmentos funiculares 5 y 6 con una sensila cada uno. Maza alrededor de 2.6 veces tan larga como ancha y más o menos tan larga como los 3 segmentos funiculares precedentes juntos. Mandíbulas, palpos labiales y maxilares como en la hembra. Ala anterior 2.8 veces tan larga como la anchura máxima de la ala.

Comentarios. Metaphycus cerealis n. sp. es similar a otras especies del género que parasitan mosquitas blancas pero difiere de todas ellas en dos características importantes: la mandíbula bidentada y la fórmula palpal 2-1. M. omega Noyes también presenta mandíbula bidentada pero su fórmula palpal es 2-2; por su parte, $M$. acapulcus Myartseva \& Ruíz y M. aleyrodis Myartseva \& Ruíz presentan mandíbulas tridentadas. Además, sólo la nueva especie tiene las alas anteriores ahumadas debajo de la vena marginal y el ovipositor 4 veces tan largo como la 
tercera válvula; las otras especies anotadas presentan alas anteriores hialinas y ovipositor menos de 3.6 veces tan largo como la tercera válvula.

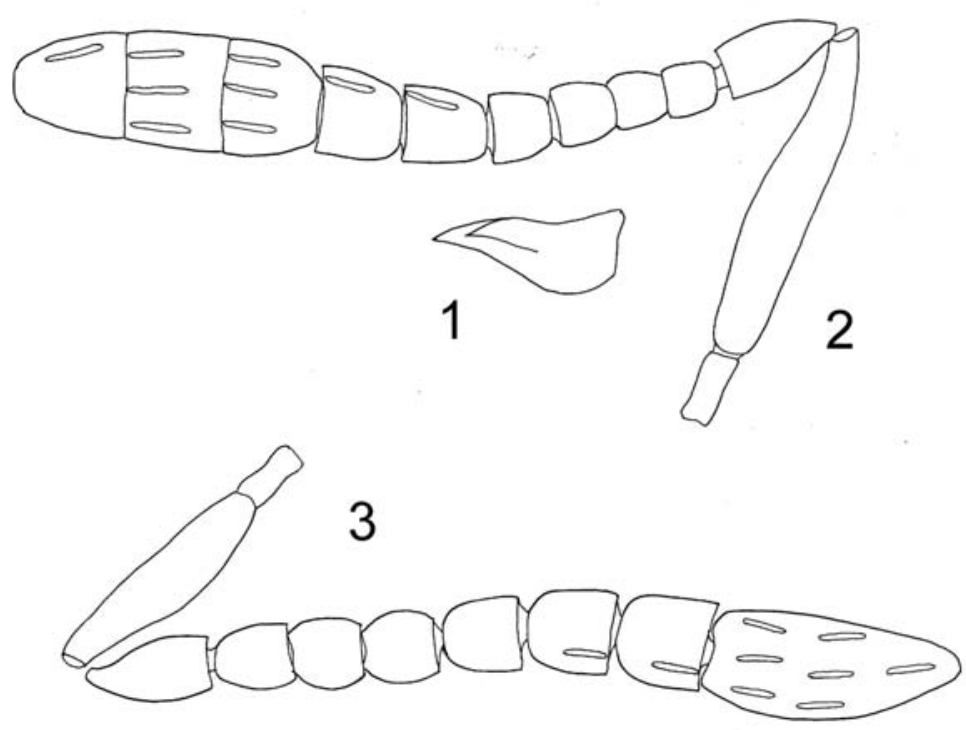

Figuras 1-3. Metaphycus cerealis, n. sp.: 1 - mandíbula, 2 - antena, hembra, 3 - antena, macho.

Todas las especies (descritas o no descritas) de Metaphycus que han sido obtenidas de mosquitas blancas, están distribuídas en la región Neotropical. La lista de especies de mosquitas blancas conocidas como hospederas de Metaphycus spp. incluye más de 14 especies (Cuadros 1 y 2). En el Cuadro 2 se enlistan las mosquitas blancas atacadas por especies de Metaphycus no identificadas.

El estudio de la morfología de la nueva especie, Metaphycus cerealis, y de otras especies conocidas (excluyendo a $M$. zdeneki y posiblemente a $M$. angustifrons - no se conoce su fórmula palpal) que atacan mosquitas blancas, muestra que estas especies pueden integrar un grupo separado en el género Metaphycus. Este grupo se caracteriza por presentar palpos maxilares de 1 ó 2 segmentos, palpos labiales raramente de 2 segmentos, líneas notaulares ausentes o sólo indicadas lateralmente, escutelo con pocos pares de setas, y la mandíbula bidentada o tridentada. Algunas otras especies de Metaphycus que normalmente atacan escamas pueden desarrollarse con éxito en aleyródidos (posiblemente también $M$. zdeneki). Se sabe que varias especies aún no descritas de Metaphycus ocasionalmente atacan mosquitas blancas en el Continente Americano (Guerrieri \& Noyes 2000). 


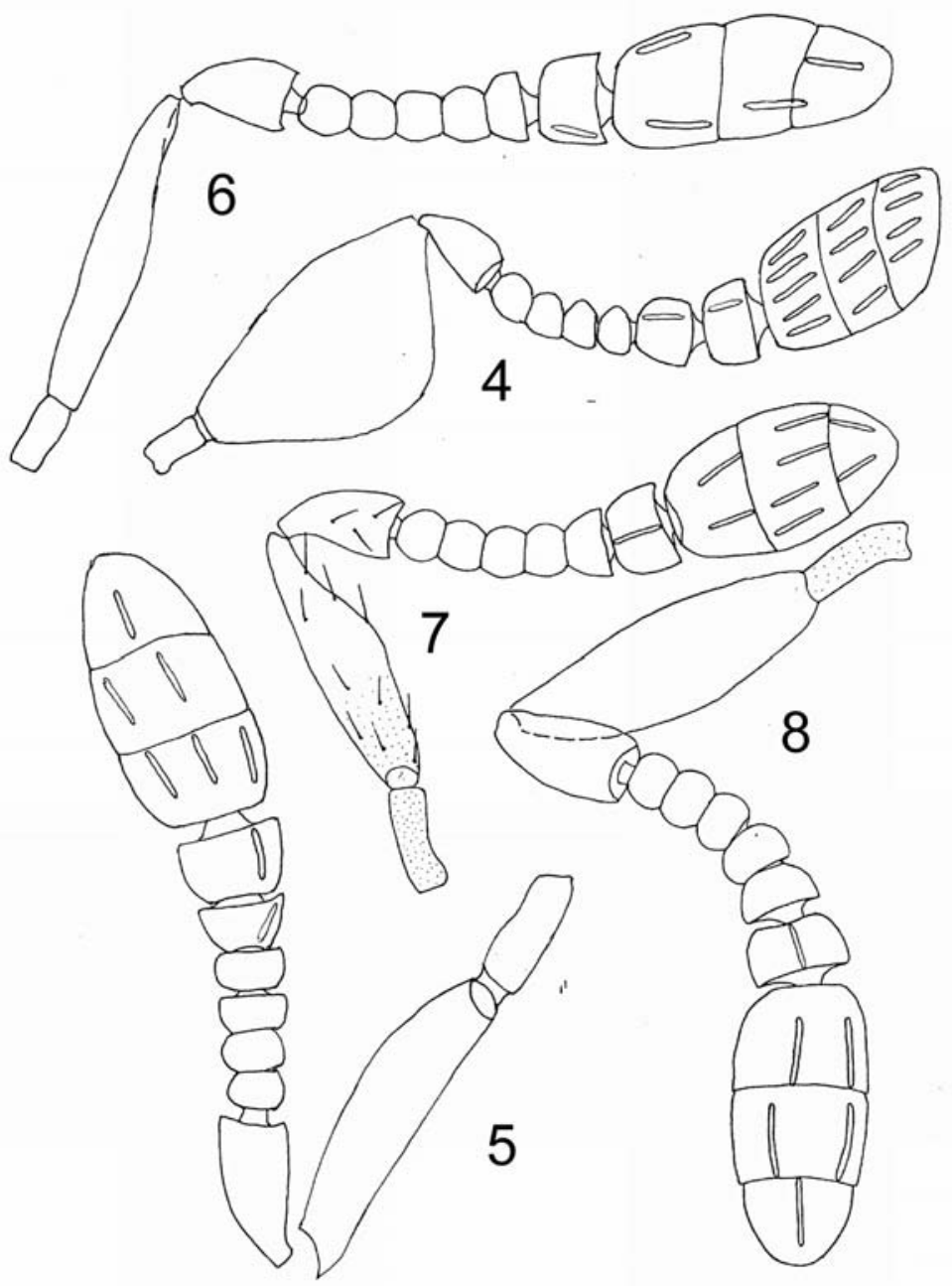

Figuras 4-8. Antenas de Metaphycus spp. (hembras): 4 - M. zdeneki, 5 - M. omega, 6 - M. troas, 7- M. acapulcus, 8 - M. aleyrodis. 
Myartseva \& Ruíz-Cancino: Nueva especie de Metaphycus Mercet (Hymenoptera: Encyrtidae) de México

Cuadro 1. Especies de Metaphycus obtenidas de mosquitas blancas en los neotrópicos.

\begin{tabular}{llll}
\hline Parasitoide & Mosquita blanca & Distribución & Referencias \\
\hline M. acapulcus & Aleurodicus sp. & México & Myartseva \& Ruíz Cancino, 2003 \\
M. aleyrodis & Tetraleurodes sp. & México & Myartseva \& Ruíz Cancino, 2002 \\
M. omega & Aleurodicus cocois & Trinidad \& & Kairo et al., 2001; Noyes, 2008 \\
& A. maritimus & Tobago, Costa & \\
& Paraleyrodes sp. & Rica, Brasil, & \\
& Aleurothrixus floccosus & Ecuador, Guyana, \\
& Dialeurodes citri & Paraguay & \\
M. troas & Trialeurodes floridensis & Costa Rica, & Noyes, 2004; Myartseva, 2006 \\
& & México & \\
M. zdeneki & Bakerius sp. & Perú & Noyes \& Lozada, 2005 \\
M. cerealis n.sp. & Aleurocybotus occiduus & México & Este trabajo \\
M. angustifrons & Aleyrodidae & Cosmopolita & Noyes, 2008 \\
\hline
\end{tabular}

Cuadro 2. Mosquitas blancas que hospedan a Metaphycus spp. en los neotrópicos.

\begin{tabular}{|c|c|c|}
\hline Especie & País & Referencias \\
\hline Aleurothrixus floccosus (Maskell) & Venezuela & Arnal et al., 2003 \\
\hline \multicolumn{3}{|l|}{ Dialeurodes sp. } \\
\hline Bemisia tabaci Gennadius & Neotrópicos & Polaszek et al., 1992 \\
\hline B. tuberculata Bondar & Colombia & Trujillo et al., 2004 \\
\hline Bemisia spp. & Venezuela & Schuster et al., 1998 \\
\hline
\end{tabular}

\section{Clave de especies de Metaphycus que parasitan mosquitas blancas en los neotrópicos (hembras)}

1. Escapo antenal principalmente negro, 1.6 a 2 veces tan largo como ancho o cerca de 2.5

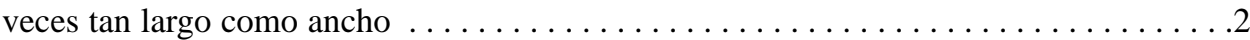

- Escapo antenal amarillo o sólo ligeramente ahumado en la base, cilíndrico, más de 3 veces

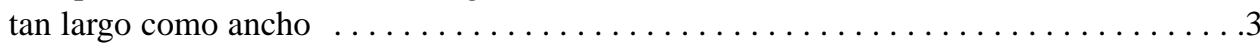

2. Escapo antenal ligeramente menos de 2 veces tan largo como ancho (Fig. 4). Ovipositor alrededor de 6.6 veces tan largo como la tercera válvula. Cabeza 3 veces tan ancha como el frontovértice . . . . . . . . . . . . . . . . . . . . . .zdeneki Noyes \& Lozada

- Escapo antenal cerca de 2.5 veces tan largo como ancho. Ovipositor alrededor de 5 veces tan largo como la tercera válvula. Cabeza 5-6 veces tan ancha como el frontovértice .... $\ldots \ldots \ldots \ldots \ldots \ldots \ldots \ldots \ldots \ldots \ldots \ldots \ldots \ldots \ldots \ldots \ldots \ldots \ldots \ldots$ ang $\ldots \ldots \ldots \ldots$ Compere 


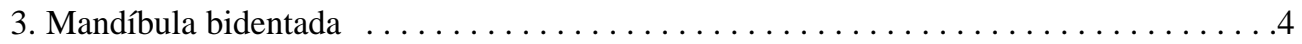

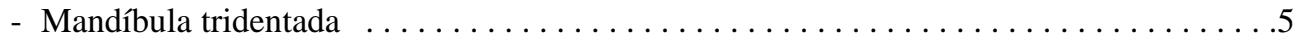

4. Fórmula palpal 2-2. Cuerpo generalmente pardo oscuro, fémur posterior pardo oscuro, ala anterior hialina. Escapo antenal cerca de 4 veces tan largo como ancho (Fig. 5). Líneas notaulares ausentes. Ovipositor ligeramente más corto que la tibia media . . . . . . . .

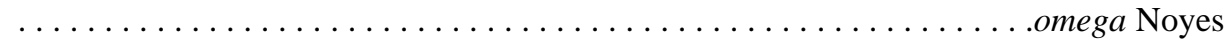

- Fórmula palpal 2-1. Cuerpo generalmente amarillo, fémur posterior amarillo, ala anterior ahumada debajo de la vena marginal. Escapo antenal 5.0-6.5 veces tan largo como ancho (Fig. 2). Líneas notaulares indicadas lateralmente. Ovipositor ligeramente más largo que la tibia media $\ldots \ldots \ldots \ldots \ldots \ldots \ldots \ldots \ldots \ldots \ldots \ldots \ldots \ldots \ldots \ldots \ldots \ldots \ldots \ldots$ cerealis $\mathrm{n} . \mathrm{sp}$.

5. Cabeza y cuerpo amarillos. Escapo antenal cerca de 4 veces tan largo como ancho (Fig. 6). Ovipositor cerca de 3 veces tan largo como la tercera válvula . . . . . . . . troas Noyes

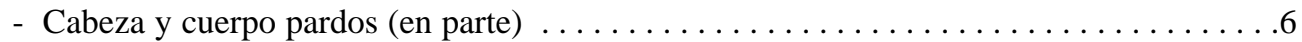

6. Escutelo con 4 pares de setas. Escapo antenal 4 veces tan largo como ancho o más (Fig. 7). Ovipositor 3.6 veces tan largo como la tercera válvula . . . acapulcus Myartseva \& Ruíz

- Escutelo con 3 pares de setas. Escapo antenal ligeramente más de 3 veces tan largo como ancho (Fig. 8). Ovipositor 2.3 veces tan largo como la tercera válvula . . . . . . . . . . .aleyrodis Myartseva \& Ruíz

AGRADECIMIENTOS. Los autores agradecen a Guadalupe Véjar-Cota (Compañía Azucarera de Los Mochis, Sinaloa, México) por la colecta de parasitoides de mosquitas blancas en gramíneas y a Edgardo Cortez-Mondaca (Escuela Superior de Agricultura del Valle del Fuerte, UAS, Sinaloa, México) por el envío de estos interesantes materiales. Al proyecto CONACYT 52587 "Avispas parasíticas de mosquitas blancas en frutales y ornamentales de Tamaulipas, México" y al proyecto PROMEP "Taxonomía y ecología de fauna y micobiota en comunidades forestales y cultivos", por su apoyo parcial para la realización de este trabajo. A los revisores de este artículo, por sus acertados comentarios.

\section{LITERATURA CITADA}

Arnal, E., A. Chávez \& F. Ramos. 2003. Parasitoides registrados en moscas blancas (Homoptera: Aleyrodidae) colectados en Venezuela durante el período 1991-2000. Entomotropica, 18(3): 183-191.

Guerrieri, E., \& J.S. Noyes. 2000. Revision of European species of genus Metaphycus Mercet (Hymenoptera: Chalcidoidea: Encyrtidae), parasitoids of scale insects (Homoptera: Coccoidea). Systematic Entomology, 25: 147-222.

Kairo, M.T.K., F. López, G.V. Pollard \& R. Hector. 2001. Biological control of the coconut whitefly, Aleurodicus pulvinatus, in Nevis. Biological News and Information, 22 (2): 45N-50N.

Myartseva, S.N. 2006. Species of the genus Metaphycus Mercet (Hymenoptera: Encyrtidae) parasitizing whiteflies (Homoptera: Aleyrodidae). Zoosystematica Rossica, 14 (2), 2005: 266.

Myartseva, S.N., \& E. Ruíz Cancino. 2002. A new species of Ooencyrtus Ashmead (Hymenoptera: Encyrtidae) reared from an aleyrodid (Homoptera) in Mexico. Zoosystematica Rossica, 11 (1): 175177.

Myartseva, S.N., \& E. Ruíz Cancino. 2003. Two species of the genus Metaphycus Mercet (Hymenoptera: Encyrtidae), parasitoids of whiteflies (Homoptera: Aleyrodidae) in Mexico. Entomología Mexicana, 2: 751-755. 
Myartseva, S.N., \& E. Ruíz Cancino. 2004. Synopsis of species of the genus Metaphycus Mercet, 1917 of Mexico (Hymenoptera: Encyrtidae) with description of new species. Russian Entomological Journal, 13 (4): 269-276.

Noyes, J.S. 2004. Encyrtidae of Costa Rica (Hymenoptera: Chalcidoidea), 2. Metaphycus and related genera, parasitoids of scale insects (Coccoidea) and whiteflies (Aleyrodidae). Memoirs of the American Entomological Institute, 73: 1-459.

Noyes, J.S. 2008. Universal Chalcidoidea Database. World Wide Web electronic publication. www.nhm.ac.uk/entomology/chalcidoids/index html (Última actualización: Mayo 2008).

Noyes, J.S., \& P.W. Lozada. 2005. Metaphycus zdeneki sp. nov. (Hymenoptera: Encyrtidae) from Peru, a parasitoid of Bakerius sp. (Hemiptera: Aleyrodidae). Acta Societatis Zoologicae Bohemoslovenicae, 69: 209-214.

Noyes, J.S., J.B. Woolley, \& G. Zolnerowich. 1997. Encyrtidae. Pp. 170-320. In: Gibson, G.A.P., J.T.Huber \& J.B. Woolley (Eds.). Annotated keys to the genera of Nearctic Chalcidoidea (Hymenoptera). NRC Research Press, Ottawa, Canada.

Ortega, A.L.D., G. Véjar C. \& V.E. Carapia. 2008. Descripción de la mosca blanca de los cereales Aleurocybotus occiduus (Russell) (Hemiptera: Aleyrodidae) y plantas hospederas en el Norte de Sinaloa. Entomología Mexicana, 7: 610-614.

Polaszek, A., G.A. Evans, \& F.D. Bennett. 1992. Encarsia parasitoids of Bemisia tabaci (Hymenoptera: Aphelinidae, Homoptera: Aleyrodidae): a preliminary guide to identification. Bulletin of Entomological Research, 2: 375-392.

Schuster, D.J., G.A. Evans, F.D. Bennett, P.A. Stansly, R.K. Jansson, G.L. Leibee, \& S.E. Webb. 1998. A survey of parasitoids of Bemisia spp. whiteflies in Florida, the Caribbean, and Central and South America. International Journal of Pest Management, 44 (4): 255-260.

Trujillo, H.E., B. Arias, J.M. Guerrero, P. Hernández, A. Bellotti, \& J.E. Peña. 2004. Survey of parasitoids of whiteflies (Homoptera: Aleyrodidae) in cassava growing regions of Colombia and Ecuador. Florida Entomologist, 87 (3): 268-273. 